\section{PWE-036 POLYETHYLENE GLYCOL PURGATIVES PRIOR TO SMALL BOWEL CAPSULE ENDOSCOPY IMPROVE DISTAL SMALL BOWEL VISUALISATION}

${ }^{1}$ David Tai*, ${ }^{1}$ Ailish Healy, ${ }^{1}$ Ryan Jennings, ${ }^{1}$ Vicky Thurston, ${ }^{1}$ Nicholas Wray, ${ }^{1}$ Reena Sidhu, ${ }^{2}$ Lawrence Hookey, 'Mark McAlindon. 'Sheffield Teaching Hospitals, Sheffield, UK; ${ }^{2}$ Kingston Health Sciences Centre, Kingston, Canada

\subsection{6/gutjnl-2019-BSGAbstracts.323}

Introduction Bowel purgatives are common practice prior to small bowel capsule endoscopy (SBCE). The use of Polyethylene glycol (PEG) laxatives is suggested to improve SB visualisation quality and diagnostic yield [Kotwal, Eur J Gastro Hep 2014]. However, this finding is not completely consistent, with other studies suggesting no clear benefit of PEG preparation over clear fluids only [Hookey, GIE 2017]. Therefore, unlike colonoscopy where the routine use of split dose bowel preparation to improve right colon cleansing has become a standard of care [Flemming, GIE 2012], the benefit and timing of purgatives prior to SBCE is unknown. We report the interim results from a clinical trial comparing the use of split dose PEG and single dose PEG against clear fluids only.

Methods Adult patients referred for SBCE were invited to participate and subject to exclusion criteria, were randomised to control (clear fluids only), single dose PEG (2L PEG at 6 am on the day of the exam) and split dose PEG (1L PEG at $7 \mathrm{pm}$ the day before and $6 \mathrm{am}$ on the day of the exam). Briefly, the mean red to green colour intensity from SBCE procedure colour bars were used to calculate a computed assessment of cleansing (CAC) scores described elsewhere [Van Weyenberg, Endoscopy 2011]. The CAC is a validated 10 point scoring system (0-10; least - most clean) used to assess small bowel visualisation quality. CAC scores are reported as mean \pm SEM and student t-tests performed to compare the means.

Results A total of 78 patients (35\% male, mean age $48 \pm 2.0$ ) were included (split $\mathrm{n}=28$, single $\mathrm{n}=24$ and control $\mathrm{n}=26$ ). Intention to treat analysis show that split dose PEG preparation results in significantly greater mean CAC score in the distal quartile $(5.58 \pm 0.16)$ of the small bowel compared to control $(4.78 \pm 0.30 ; p=0.02)$. No differences in the overall, first, second and third quartile CAC score of the small bowel was found between control and PEG groups. Complete ingestion of PEG preparation occurred in $88 \%$ and $77 \%$ of single vs split dose $(p=0.33)$. Subgroup analysis showed that amongst patients that completed preparation successfully, those randomised to both split dose $(5.52 \pm 0.20 ; \mathrm{p}=0.01)$ and single dose $(5.64 \pm 0.18, p=0.003)$ PEG had a significantly greater mean CAC score in the distal quartile of the small bowel compared to control $(4.58 \pm 0.28)$.

Conclusions The use of split dose laxatives improves the visualisation quality of the distal small bowel. Initial results may suggest that the PEG dose in the morning of the procedure improves distal quartile visualisation.

\section{PWE-037 DIAGNOSIS AND MANAGEMENT OF BILE ACID DIARRHOEA: UK CONSENSUS SURVEY OF EXPERT OPINION AND PRACTICE}

${ }^{1} J u l i a n$ Walters*, ${ }^{2}$ Ramesh Arasaradnam, ${ }^{3}$ Jervoise Andreyev, UK Bile Acid Related Diarrhoea Network. 'Imperial College London, London, UK; ${ }^{2}$ University Hospital Coventry, Coventry, UK; ${ }^{3}$ Lincoln County Hospital, Lincoln, UK

\subsection{6/gutjnl-2019-BSGAbstracts.324}

Introduction Bile acid diarrhoea (BAD), including bile acid malabsorption (BAM), causes a variety of digestive symptoms and is increasingly recognised, although diagnostic rates and management vary considerably. The UK Bile Acid Related Diarrhoea Network (UK-BARDN), established in 2017, conducted a survey of current practice to provide a review of expert opinion and guidance on diagnosis and management. Methods An on-line survey was sent at the end of 2018 to 21 clinical members of UK-BARDN, who had all published research on BAD.

Results A response rate of $100 \%$ was obtained. 95\% were NHS Consultants; $85 \%$ estimated they had diagnosed over 50 patients with the condition. BAD was the terminology preferred by $57 \%$, with another $29 \%$ using BAD or BAM depending on the clinical circumstances. Primary and secondary were the preferred terms to classify the different causes.

A wide range of presenting symptoms and associated conditions were recognised. SeHCAT was the preferred diagnostic test, with a therapeutic trial the second choice. Access to SeHCAT by GPs was thought appropriate by $50 \%$, with greater availability of specific blood tests in hospitals. SeHCAT would usually be requested $(>70 \%)$ in patients who met the diagnostic criteria for functional diarrhoea, IBS-D, or post-cholecystectomy diarrhoea, and sometimes be requested $(>30 \%)$ in other types of IBS, and in Crohn's disease with ileal resection and negative inflammatory markers, where a therapeutic trial was also commonly used.

Treatment with a bile acid sequestrant (BAS) would always be given if SeHCAT was $<5 \%$, usually if $5-15 \%$, and sometimes if $15-20 \%$. Expected response rates were $>70 \%$, falling to $30 \%$ in these groups. Colestyramine was the usual first line BAS, with starting doses varying between $2 \mathrm{~g}$ od and $4 \mathrm{~g}$ bd. Colesevelam was also used. There was a slight preference to give the drug at bedtime. Warnings about drug interactions were usual. In patients who had an incomplete response, increasing the dose, changing to an alternative BAS, increasing use of drugs such as loperamide, and advice on a low fat (40 $\mathrm{g} / \mathrm{d}$ ) diet were commonly recommended. During follow-up, annual review by a specialist or GP, patient support groups, dietetic and pharmacist review, and monitoring of blood vitamins and lipids were given varying importance. To improve the overall patient experience, greater recognition by various professional groups and in the popular press, with improved diagnosis and drugs, were considered important.

Conclusions This expert consensus provides a basis for current best practice in the diagnosis and management of patients with BAD. 